\title{
Bifurcation and Chaos in a Complex Model of Dissipative Medium
}

\author{
Marius-F. Danca \\ Department of Mathematics, "Tehnofrig" College \\ 3400 Cluj-Napoca, Romania \\ Guanrong Chen \\ Department of Electronic Engineering \\ City University of Hong Kong, P. R. China
}

\begin{abstract}
In this paper, we carefully re-examine the chaotic RF model, first studied by Rabinovich \& Fabrikant [1979], and found many new and rich complex dynamics of the model that were mostly not reported before. The chaotic RF model has proved to be a great challenge to classical numerical methods, in the sense that most classical numerical methods have not been very successful in studying the complex dynamics of this special RF model. Therefore, in this paper, we develop and apply a special numerical method, the Local Iterative Linearization (LIL) method, along with a special Turbo Pascal code based on this accurate LIL algorithm, for a careful numerical study of this complex RF model. Many interesting new findings are summarized and reported in this paper.
\end{abstract}

Keywords: bifurcation, chaos, iterative linearization method

\section{Introduction}

Rabinovich \& Fabrikant [1979] studied the following dynamical system (named the RF model hereafter):

$$
\begin{aligned}
& \dot{x_{1}}=x_{2}\left(x_{3}-1+x_{1}^{2}\right)+a x_{1}, \\
& \dot{x_{2}}=x_{1}\left(3 x_{3}+1-x_{1}^{2}\right)+a x_{2}, \\
& \dot{x_{3}}=-2 x_{3}\left(b+x_{1} x_{2}\right),
\end{aligned}
$$

where the constant parameters $a, b>0$. This system models the stochasticity arising from the modulation instability in a non-equilibrium dissipative medium. Some qualitative analysis and numerical dynamics have been reported [Rabinovich \& Fabrikant, 1979]. 
The aim of this paper is to extend the numerical experiments reported in [Rabinovich \& Fabrikant, 1979]. This particular system has proved to be a great challenge to classical numerical methods; in fact, most classical numerical methods have not been very successful in studying the complex dynamics of the RF model (1) to date.

Therefore, in this paper, we developed and applied a special numerical method, which is an implicit multistep numerical method [Danca, 1997], called the Local Iterative Linearization (LIL) method [Colosi et al., 1999] and is given in the Appendix.

The system has been implemented via a Matlab-Simulink circuit, as shown in Figure 1.

The complex dynamical behaviors of the system, obtained by our LIL simulation, are shown in Figures 3-35. These figures will be illustrated in detail one by one below, but at this point a quick glance reveals some very interesting new periodical and chaotic phenomena from the RF model (1), which are mostly not reported in [Rabinovich \& Fabrikant, 1979].

This paper is organized as follows: In Section 2, some analytical results about the equilibrium points, Jacobian, and divergence etc. are derived; in Section 3, LIL numerical results about stabilities, bifurcation diagrams, phase portraits, Poincaré sections, first return maps, and times series etc. are presented; conclusions are given in Section 4, along with some discussions; finally in the Appendix, the LIL algorithm for numerical integration of ordinary ODEs is described.

\section{System Equilibrium Points}

The equilibrium points of the RF model (1) are

$$
\begin{aligned}
& X^{*}(0,0,0), \\
& X_{1,2}^{*}\left( \pm x_{-},-b / x_{-}, 1-\left(1-\frac{a}{b}\right) x_{-}^{2}\right), \\
& X_{3,4}^{*}\left( \pm x_{+},-b / x_{+}, 1-\left(1-\frac{a}{b}\right) x_{+}^{2}\right)
\end{aligned}
$$

with

$$
x_{ \pm}=\sqrt{\frac{1 \pm \sqrt{1-a b(1-3 a / 4 b)}}{2(1-3 a / 4 b)}}
$$

The existence domain of $X_{1,2,3,4}^{*}$ is drawn in Figure 2.

All the equilibrium points are hyperbolic. The origin is an equilibrium point that does not depend on the parameters $a, b$.

The right-hand side of the RF model (1), $f=\left(f_{1}, f_{2}, f_{3}\right)^{t}: \mathbb{R}^{3} \rightarrow \mathbb{R}^{3}$, is a smooth real-valued function with the following symmetry:

$$
\begin{aligned}
& f_{1}\left(-x_{1},-x_{2}, x_{3}\right)=-f_{1}\left(x_{1}, x_{2}, x_{3}\right) \\
& f_{2}\left(-x_{1},-x_{2}, x_{3}\right)=-f_{2}\left(x_{1}, x_{2}, x_{3}\right) \\
& f_{3}\left(-x_{1},-x_{2},-x_{3}\right)=-f_{3}\left(x_{1}, x_{2}, x_{3}\right)
\end{aligned}
$$


which can be observed in the disposal of equilibrium points or limit cycles.

The system Jacobian, which will be evaluated at the equilibrium points later, is given by

$$
J=\left(\begin{array}{ccc}
2 x y+a & x^{2}+z-1 & y \\
-3 x^{2}+3 z+1 & a & 3 x \\
-2 y z & -2 x z & -2(x y+b)
\end{array}\right)_{X^{*}}
$$

It is clear that this Jacobian is rather complicated; therefore, analytically studying the stability of the equilibrium points $X_{1,2,3,4}^{*}$ is not an easy task.

Observe that

$$
\operatorname{div} f\left(x_{1}, x_{2}, x_{3}\right)=\sum_{i=1}^{3} \frac{\partial}{\partial x_{i}} f\left(x_{1}, x_{2}, x_{3}\right)=2(a-b)
$$

It follows that the system is dissipative for $a<b$, because using the Liouville formula we have $V(t)=V(0) e^{t d i v f(x)}=V(0) e^{2(a-b) t}$, which means that at time $t$, an arbitrary volume element $V$ enclosed by some surface $S$ in the phase space $\mathbb{R}^{3}$, is contracting if $a<b$. Hence, for $a<b$, the system is characterized by the appearance of attractors in the phase space.

\section{$3 \quad$ Stability and Bifurcation Analysis}

We have observed that the system behavior is very sensitive to parameter $b$ but not as so to $a$. Hence, we fixed $a=0.1$ and let $b$ be varied. It is easy to see that the system characteristic polynomials at $X_{1,2}^{*}$ and $X_{3,4}^{*}$ are similar, due to the symmetry of the RF model (1).

Because the extreme sensitivity, numerical integration of the system for $b>$ 1.3 and $b<0.13$ turns out to be very difficult. We therefore chose for $b$ the range $\left(b_{\min }, b_{\max }\right)$, with $b_{\min }=0.13$ and $b_{\max }=1.3$. For the region of interest defined by $b \in\left(b_{\min }, b_{\max }\right)$ and $a=0.1$, the system is dissipative. However, note that some results were found for $b \notin\left(b_{\min }, b_{\max }\right)$ and $a \neq 0.1$.

\subsection{Stability of system equilibrium points}

Let us now consider, in the three-dimensional space $(z b \lambda)$ for a specified point $X^{*}$, the following surfaces:

$$
S_{X^{*}}(b, \lambda)=\left\{P_{X^{*}}(\lambda)\right\}=\left\{|\lambda I-J|_{X^{*}}, \text { for } b \in\left(b_{\min }, b_{\max }\right), \lambda \in \mathbb{R}\right\}
$$

which are the set of all system characteristic polynomials $P_{X^{*}}$ at $X_{1,2,3,4}^{*}$ for $b \in\left(b_{\min }, b_{\max }\right)$.

The intersection of surfaces $z=S_{X^{*}}(b, \lambda)$ with the plane $z=0$ gives the set of eigenvalues (i.e., the roots of characteristic equations $P_{X^{*}}(\lambda)=0$ for $\left.b \in\left(b_{\min }, b_{\max }\right)\right)$, which are disposed on the following curves:

$$
\Lambda(b, \lambda)=\left\{(b, \lambda), P_{X^{*}}(\lambda)=0, b \in\left(b_{\min }, b_{\max }\right)\right\}
$$


In order to obtain the graph of a specific characteristic polynomial, $P_{X^{*}}$ for some $b=\bar{b} \in\left(b_{\min }, b_{\max }\right)$, we let the surface $S_{X^{*}}$ intersect with the plane $b=\bar{b}$.

The equilibrium point $X_{0}^{*}$ have the following characteristic equation:

$$
|\lambda I-J| \equiv\left(\lambda^{2}-2 a \lambda+a^{2}+1\right)(\lambda+2 b)=0
$$

with the eigenvalues

$$
\lambda_{1}=-2 b<0, \text { and } \lambda_{2,3}=a \pm i
$$

Therefore, $X_{0}^{*}$ is a saddle-focus.

Next, consider $X_{1,2}^{*}$. At these points, graphs of $S_{X_{1,2}^{*}}$ and $P_{X_{1,2}^{*}}$ are plotted in Figure 3. In the background, the projections of the characteristic polynomials $P_{X_{1,2}^{*}}$ for different values of $b$ are represented. The real eigenvalue $\lambda_{1}$ is positive for any $b$ (see the curve $\Lambda$ ). The two other eigenvalues, $\lambda_{2,3}$, are imaginary conjugate with a negative real part (Figure 4 ). The entries used to obtain this plot were determined numerically. The existence of the complex eigenvalues, and also the existence of the only real eigenvalue, can be deduced from the plot of $S_{X_{1,2}^{*}}$, which indicates a monotonically decreasing map of variable $\lambda$ since the derivative $S_{X_{1,2}^{*}}^{\prime}$ is negative for all values of $b$ (Figure 5). Hence, we can conclude that $X_{1,2}^{*}$ are saddle-foci.

Hence, for any $b \in\left(b_{\min }, b_{\max }\right), X_{0}^{*}$ and $X_{1,2}^{*}$ are unstable equilibrium points and, because of the complex eigenvalues, the system trajectories starting near $X_{0,1,2}^{*}$ spiral away from these points.

For $X_{3,4}^{*}$, the characteristic equations

$$
P_{X_{3,4}^{*}}(\lambda)=\left.S_{X_{3,4}^{*}}\right|_{b=\bar{b}}=0, \quad \bar{b} \in\left(b_{\min }, b_{\max }\right)
$$

have two complex eigenvalues, $\lambda_{1,2}$, and a real one $\lambda_{3}$, numerically determined. Actually, for the first values of $b$, close to $b_{\min }$, the complex eigenvalues have a very small imaginary part (in an order of $10^{-4} \div 10^{-3}$ ). The real eigenvalue, on the other hand, can be seen from Figures 6 and 7, which indicate that it is negative. The existence of the complex eigenvalues can also be deduced analytically from the monotonicity of $P_{X_{3,4}^{*}}$. The real component of $\lambda_{1,2}$ is negative for $b \in\left(b_{\min }, b^{*}\right)$, where $b^{*} \simeq 1.025$, and is positive for $b \in\left(b^{*}, b_{\max }\right)$, as can be seen from Figure 8. Therefore, $X_{3,4}^{*}$ are stable for $b \in\left(b_{\min }, b^{*}\right)$ (stable node - stable focus) and saddle for $b \in\left(b^{*}, b_{\max }\right)$ (stable node - unstable focus). Due to the existence of complex eigenvalues, the system trajectories spiral around these equilibrium points too.

The above observations on the stability of equilibrium points are summarized in Table 1.

\begin{tabular}{|c|c|c|}
\hline$b$ & $b \in\left(b_{\min }, b^{*}\right)$ & $b \in\left(b^{*}, b_{\max }\right)$ \\
\hline$X_{0}^{*}$ & unstable & unstable \\
\hline$X_{1,2}^{*}$ & unstable & unstable \\
\hline$X_{3,4}^{*}$ & stable & unstable \\
\hline
\end{tabular}

Table 1. Stability of equilibrium points of system (1) for $b \in\left(b_{\min }, b_{\max }\right)$ 


\subsection{Bifurcation diagrams of system dynamics}

The bifurcation diagrams of the RF model (1), obtained by the LIL numerical algorithm, are depicted in Figures 9. To obtain these diagrams, we plotted the maximum of the state variable as a function of the parameter $b$.

As can be seen from the diagrams, there are some switching between the branches of the equilibrium points. This is due to the strong dependence of the states on initial conditions. Also, for some values of $b$, there are some "parasites" (which must not be interpreted as indicating some chaotic motions) and this is likely due to the weak attractiveness of the equilibrium points, and/or the influence of neighboring equilibrium points. This phenomenon seems to be independent of the integration step size and the number of iterations. Although these diagrams are useful for analyzing the equilibrium points, they may lead to false prediction of chaos therefore must be used with precautions.

The graph of $x_{3 \max }$ shows some windows in which the diagram is quite similar to those of the logistic map. Hence, we may expect to find, at least in the direction $x_{3}$, period-doubling bifurcations, chaos, band merging, windows of periods 3 and 5 and veils.

Next, we follow the bifurcation diagram for $x_{3}$ and try to use the most significant values of $b$ to present the phase portraits and time series, whereas if necessary the Poincaré section, power spectrum, first return map, and histogram are presented.

\section{Remarks:}

a) Due to some integration problems encountered when using Matlab, we have chosen those cases to study for which both LIL and Matlab could be used. The power spectra and histograms were obtained using the analog computer circuit (see Figure 1) but only as an orientated direction.

b) Due to the uncertainty of the numerical results, the chaotic or strange attractors is considered only as a "possible" outcome. The attractors were considered "strange" following the argument of resemblance to strange attractor.

In the computer simulations by $t_{0}$ we mean the start of the drawing, while $m$ represents the backward integration steps used by LIL (see Appendix). We focus on the trajectories related to $X_{3,4}^{*}$ with $b$ as the key parameter, while $X_{0}^{*}$ and $X_{1,2}^{*}$ are unstable and surrounded by limit cycles (see, e.g., Figures 10 and 11):

1) $b=b_{1}=0.14$ : A periodic motion was found $\left(C_{1}\right.$ in Figure 12$)$. The points $X_{3,4}^{*}$ have extremely weak attractivity (as indicated by the large value of $t_{\max }$, necessary to reach the points).

2) $b=b_{2}=0.19$ : In addition to the above-mentioned periodic cycle, we found that $t_{\max }$, necessary to reach $X_{3,4}^{*}$, is significatively decreased (Figure 13).

3) $b=b_{3}=0.2715: C_{1}$ may transform into chaotic motion (Figure 14). To verify this, we computed the Lyapunov exponents: $\lambda_{1}=-0.431, \lambda_{2}=$ 
$-0.003, \lambda_{3}=0.091$, and the first return map, Poincare section, power spectra (Figures 15). The points $X_{3,4}^{*}$ are attractive.

4) $b=b_{4}=0.285$ : The periodic cycle $C_{1}$ is again stable, and is growing with its period, while $X_{3,4}^{*}$ maintain their stability (Figure 16).

5) $b=b_{5}=0.2876$ : Again, a chaotic attractor appears (Figure 17). Now, from Figure 18, we can see a quasi-periodic motion (with Lyapunov exponents $\lambda_{1}=-0.441, \lambda_{2}=0.064, \lambda_{3}=0.002$ ).

6) $b=b_{6}=0.98$ : The first strange attractor appears (Figure 19). The chaotic behavior can be predicted from Figures 20 .

7) $b=b_{7}=1.03$ : Once again, the stable limit cycle $C_{1}$, which increases as its period increases, together with the stable $X_{3,4}^{*}$, are obtained (Figure 21).

8) $b=b_{8}=1.08$ : After a Hopf bifurcation at $b^{*} \simeq 1.025$, points $X_{3,4}^{*}$ transform into two stable limit cycles, $C_{2,3}$, near $C_{1}$ (Figure 22).

9) $b=b_{9}=1.12: C_{1}$ is stable and $C_{2,3}$ remain to be period-one limit cycles (Figure 23).

We could not find $C_{2,3}$ for $b \in(1.13,1.2)$. It seems that $C_{1}$ has a stronger attractivity than $C_{2,3}$, and any trajectory moving to $C_{2,3}$ is "absorbed" by $C_{1}$.

10) $b=b_{10}=1.21$ : A chaotic double-scroll motion appears. Again, the three attractors, $C_{1}$ and $C_{2,3}$, seem to be indistinctive (Figure 24). In Figures 25 , the power spectra, Poincaré sections, histograms, and first return maps are all presented.

11) $b=b_{11}=1.215$ : The above-mentioned double-scroll attractor separates (Figure 26). The chaotic behavior can be seen from Figures 27.

12) $b=b_{12}=1.225$ : Two periodic attractors appear (Figure 28).

In Figures 29-32, other possible periodic motions for $b \notin\left(b_{\min }, b_{\max }\right)$ and $a \neq 0.1$ are presented, while in Figure 33 an interesting strange attractor for $a$ and $b<0$, different to those obtained for $a, b>0$, was obtained.

\section{Conclusions and discussions}

In this paper, we have carefully examined the chaotic RF model, first studied by Rabinovich \& Fabrikant [1979], and found many new and rich complex dynamics of the model that were mostly not reported in [Rabinovich \& Fabrikant, 1979].

It is well known that because of the sensitive dependence on initial conditions, a chaotic system tends to amplify, often exponentially, tiny initial errors [Chen \& Dong, 1998]. These kind of errors could be amplified to so large that it 
is almost impossible to draw mathematically rigorous conclusions based on numerical simulations. A typical case can be seen from Figures 34, wherefrom one can deduce that the attractor size along the $x_{3}$-axis increases significatively as the step-size decreases. This problem has been noticed for a long time, and has promoted a useful theory called "shadowing," namely, the existence of a true orbit nearby a numerically computed approximate orbit [Coomes et al. 1995].

We have also found that the strong dependence on the step-size produces, for some values of $b$ and with same initial conditions, some totally different attractors (see Figures 30 and 35).

The chaotic RF model has proved to be a great challenge to classical numerical methods; in fact, most classical numerical methods have not been very successful in studying the complex dynamics of this special RF model. Therefore, in this paper, we have developed and applied a special numerical method, the Local Iterative Linearization (LIL) method, for a more careful numerical study of this complex RF model. All computer test results and graphical plots given in this paper were carried out by using a special Turbo Pascal code based on our accurate LIL algorithm; therefore, they are very reliable.

\section{Acknowledgments}

The authors thank Miguel Romera and Fausto Montoya for their helpful suggestions regarding Matlab implementations. The second author was supported by the Hong Kong Research Grants Council under the CERG grant 1004/02E.

\section{References}

[1] Chen, G. \& Dong, X. [1998] From Chaos to Order: Methodologies, Perspectives and Applications, Singapore: World Scientific.

[2] Colosi, T., Raica, P., Nascu, I., Codreanu, S. \& Szakács, E. [1999] Local Iterative Linearization Method for Numerical Modeling and Simulation of Lamped and Distributed Parameter Processes, Casa Cãrtii de Stiintã, Cluj-Napoca.

[3] Coomes, B. A., Koçak, H. \& Palmer, K. J. [1995] "Rigourous computational shadowing of orbits of ordinary differential equations," Numerische Mathematik, 69:401-421.

[4] Danca, M.-F., PhD. Thesis (in Romanian), Technical University of Cluj-Napoca, 1997.

[5] Rabinovich, M. I. \& Fabrikant, A. L. [1979] Stochastic self-modulation of waves in nonequilibrium media, J. E. T. P. (Sov.) 77:617-629. 


\section{Appendix}

Consider the Cauchy initial value problem:

$$
\dot{x}=f(t, x), x\left(t^{0}\right)=x^{0},
$$

where $f: I \times \mathbb{R}^{n} \rightarrow \mathbb{R}^{n}$ is a single valued real map of class $C^{m}\left[\mathbb{R}^{n}\right], m \geq 1$, $I=\left[t_{0}, T\right], T>0, x^{0} \in \mathbb{R}^{n}$.

Let $N=\left(T-t_{0}\right) / h$, where $h$ is the step-size. Then the LIL (Local Iterative Linearization) implicit multistep method, applied on the equidistant grids $t_{0}<$ $t_{1}<\ldots<t_{N}=T, t_{k}=t_{0}+k h, k=1,2, \ldots, N$, is the following:

$$
x^{k}=u^{k} h+v^{k}, k=1,2, \ldots, N
$$

with

$$
u^{k}=\frac{\sum_{i=0}^{m} \sigma_{0 i} f^{k-i}}{\sigma_{10}}, v^{k}=\frac{\sum_{i=1}^{m} \sigma_{1 i} x^{k-i}}{\sigma_{10}},
$$

where $f^{k-i}=f\left(t_{k}, \sum_{i=1}^{m} \varepsilon_{m i} x^{k-i}\right), k=1,2, \ldots, N, m$ is the number of backward steps. The coefficients $\sigma$ and $\varepsilon$ for $m=3$ are drawn from Tables 2 and 3 . The most convenient case (CPU time versus accuracy) is, as in the present paper, $m=3$. The error in this case is of order $h^{4}$ (see [Danca, 1997; Colosi et al., 1999] for a more comprehensive description of the LIL method, convergence proofs and its application examples).

\begin{tabular}{|c|c|c|c|}
\hline$\sigma_{00}$ & $\sigma_{01}$ & $\sigma_{02}$ & $\sigma_{03}$ \\
\hline $13 / 12$ & $-5 / 24$ & $1 / 6$ & $-1 / 24$ \\
\hline
\end{tabular}

\begin{tabular}{|c|c|c|c|}
\hline$\sigma_{10}$ & $\sigma_{11}$ & $\sigma_{12}$ & $\sigma_{13}$ \\
\hline $15 / 8$ & $-25 / 8$ & $13 / 8$ & $-3 / 8$ \\
\hline
\end{tabular}

Table 2 Coefficient $\sigma$

\begin{tabular}{|c|c|c|}
\hline$\varepsilon_{31}$ & $\sigma_{32}$ & $\sigma_{33}$ \\
\hline 3 & -3 & 1 \\
\hline
\end{tabular}

Table 3 Coefficients $\varepsilon$ and $\sigma$ 